\title{
Distribution and congener profiles of short-chain chlorinated paraffins in indoor/outdoor glass window surface films and their film-air partitioning in Beijing, China
}

\author{
Wei Gao ${ }^{\mathrm{a}}$, Jing $\mathrm{Wu}^{\mathrm{a}}$, Yawei Wang ${ }^{\mathrm{a}, \mathrm{b}, \mathrm{c}, *}$, Guibin Jiang ${ }^{\mathrm{a}}$ \\ a State Key Laboratory of Environmental Chemistry and Ecotoxicology, Research Center for Eco-Environmental Sciences, Chinese Academy of Sciences, Beijing \\ 100085, China \\ ${ }^{\mathrm{b}}$ Institute of Environment and Health, Jianghan University, Wuhan 430056, China \\ ${ }^{c}$ University of Chinese Academy of Sciences, Beijing 100049, China
}

\section{H I G H L I G H T S}

- High levels of SCCPs were found both in the window surface films and in indoor air.

- Congener group abundance profiles varied between indoor and outdoor films.

- The relationship of different building characteristics and SCCPs distributions were studied.

- Film-air partitioning study of SCCPs suggested that indoor gas-phase SCCPs are related to their corresponding film levels.

\section{A R T I C L E I N F O}

\section{Article history:}

Received 9 July 2015

Received in revised form 18 September 2015

Accepted 19 September 2015

Available online 23 October 2015

Handling editor: Jacob de Boer

Keywords:

Short chain chlorinated paraffins

Organic films

Film-air partitioning

\begin{abstract}
A B S T R A C T
Short-chain chlorinated paraffins (SCCPs) are a group of n-alkanes with carbon chain length of 10-13. In this work, paired indoor/outdoor samples of organic films on window glass surfaces from urban buildings in Beijing, China, were collected to measure the concentrations and congener distributions of SCCPs. The total SCCP levels ranged from $337 \mathrm{ng} / \mathrm{m}^{2}$ to $114 \mu \mathrm{g} / \mathrm{m}^{2}$, with total organic carbon (TOC) normalized concentrations of $365 \mu \mathrm{g} / \mathrm{m}^{2}-365 \mathrm{mg} / \mathrm{m}^{2}$. Overall, the concentrations of SCCPs on the interior films were higher than the concentrations on the exterior films, suggesting an important indoor environmental exposure of SCCPs to the general public. A significant linear relationship was found between the SCCP concentrations and TOC, with a correlation coefficient of $\mathrm{R}=0.34(p<0.01)$. A film-air partitioning model suggests that the indoor gas-phase SCCPs are related to their corresponding window film levels.
\end{abstract}

(c) 2015 Elsevier Ltd. All rights reserved.

\section{Introduction}

Chlorinated paraffins (CPs), which are also referred to as polychlorinated n-alkanes, are semi-volatile chemicals (SVOCs) that have been used in large quantities in commercial products for several decades. Commercial CP mixtures can be divided into different categories based on their carbon chain length: short-chain (SCCPs, $\left.\mathrm{C}_{10}-\mathrm{C}_{13}\right)$, medium-chain $\left(\mathrm{C}_{14}-\mathrm{C}_{17}\right)$, and long-chain chlorinated paraffins $\left(\mathrm{C}_{20}-\mathrm{C}_{30}\right)$ (Muir et al., 2000). Among them, SC-

\footnotetext{
* Corresponding author. State Key Laboratory of Environmental Chemistry and Ecotoxicology, Research Center for Eco-Environmental Sciences, Chinese Academy of Sciences, P.O. Box 2871, Beijing 100085, China.

E-mail address: ywwang@rcees.ac.cn (Y. Wang).
}

CPs draw more attention because of their higher adverse effects (Tomy et al., 1998; Feo et al., 2009; De Boer, 2010) on the environment and human health and their potential to bioaccumulate and travel throughout the atmosphere (Krogseth et al., 2013; Ma et al., 2014). These contaminants have been included in regulatory programs from the US Environmental Protection Agency and the European Union (ECD, 2000) and now are candidates to be classified as persistent organic pollutants (POPs) under the Stockholm Convention. It is still impossible for prohibition of these products in recent future due to the lack of substitutes (De Boer, 2010) and the challenges in CPs analysis (Van Mourik et al., 2015).

China is the largest CPs producing country in the world (De Boer, 2010). Recent work has indicated that SCCPs have various anthropogenic origins and are ubiquitous in metropolitan areas in China (Yuan et al., 2012; Chen et al., 2011). Our recent study on 
the gas-particle partitioning of SCCPs in the atmosphere in Beijing (Wang et al., 2012) showed that the concentrations of SCCPs in air were significantly higher than other POPs, such as halogenated dioxins (PCDD), polychlorinated biphenyls (PCB), and polybrominated biphenyl ethers (PBDE), etc.

Urbanization gives a sharp rise of impervious surfaces in urban areas such as windows, roadways, and the exterior of buildings. Some researchers have noted that organic films can be formed through partitioning on these surfaces and have a significant influence on the transportation and fate of SVOCs in urban environments (Law and Diamond, 1998; Diamond et al., 2000; Gingrich et al., 2001). The deposition of SVOCs and wash-off make organic films both a sink and source for various pollutants. A wide range of chemicals have been found in the surface films, including POPs (e.g., polycyclic aromatic hydrocarbons (PAHs), polychlorinated biphenyls (PCBs), polybrominated diphenyl ethers (PBDEs)) (Pan et al., 2012; Wu et al., 2008; Li et al., 2010; Butt et al., 2004; Unger and Gustafsson, 2008), heavy metals (e.g., $\mathrm{Hg} \mathrm{Ag} \mathrm{Cr}$ ) ( $\mathrm{Li}$ et al., 2010; Liu et al., 2003a,b), and biogenic chemicals (e.g., polar organic compounds) (Lam et al., 2005; Liu et al., 2003a,b; Fridén et al., 2011).

The objective of this work is to study the concentrations and congener profiles of SCCPs in paired (indoor/outdoor) window glass film and in indoor air samples collected from office buildings, residential houses, and dormitories in Beijing, China. The film-air partitioning model is used to investigate the film-air partitioning mechanisms of SCCPs between the windows organic films and indoor air and evaluate the possibility of window organic films to indicate the concentrations of SCCPs in indoor air. To our knowledge, no investigation on SCCPs levels on window glass surfaces films has been performed previously. Since the data on the SCCPs in indoor environment are very scarce, the results of this work help us to better understand the exposure levels of SCCPs to the general population via indoor environments in metropolitan areas.

\section{Method section}

\subsection{Sample collection}

A total of 114 samples were collected at 18 urban sites and one suburban site (Fig. 1) from November 2013 to February 2014 in Beijing, including nine residential houses, four dormitories and six office buildings. The samples were collected in pairs (i.e., indoor/outdoor window glasses) and the sampling sites cover differ-

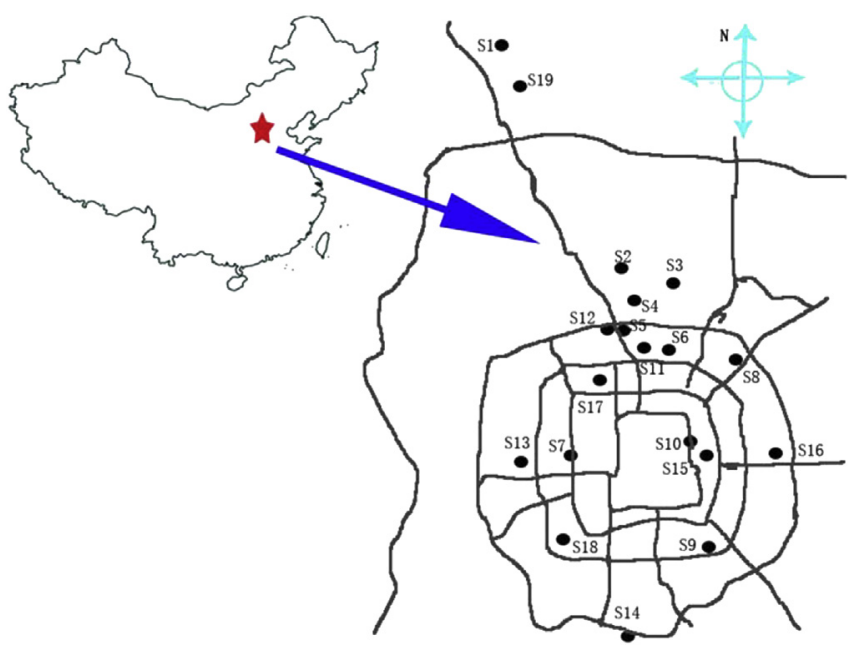

Fig. 1. Locations of the 19 sampling sites that organic films were collected on indoor/outdoor window surfaces in Beijing. ent functional areas. At each site at least one paired samples was collected and, if available, windows of different facings and at different height were sampled. The detailed information of all samples is provided in Table S1 of the Supporting Information (SI). The sampling was performed in the winter season to reduce the effects of heavy rain wash-off. Before sampling, the windows had not been cleaned for at least three months to ensure that organic films had the opportunity to form on these surfaces. The indoor air was also sampled at 11 concurrent sites (S2, S4, S5, S6, S8, S12, S14, S15, and S17-S19) out of the 19 sampling sites. Samples were collected using polyurethane form materials (PUF) passive air samplers during the entire film growth period to study the possible film-air partition of SCCPs in indoor environments and the SCCPs concentrations and congener group profiles.

The film sampling method was based on previous work with some minor modifications (Diamond et al., 2000; Li et al., 2010). Kim wipes (Kimberly-Clark, USA) with dichloromethane (DCM, HPLC grade) were used to wipe the window twice to collect the organic films for SCCP analysis. The sampling area measured approximately $0.1 \mathrm{~m}^{2}$. Before sampling, the Kim wipes were extracted using accelerated solvent extraction (ASE350, Dionex, USA) with a $1: 1$ (volume to volume) mixture of dichloromethane and hexane and then dried, weighed, wrapped with aluminum foil and placed in zipper bags to avoid contamination. Windows were sampled to within $10 \mathrm{~cm}$ of the window edge to avoid interferences from paints, exterior caulking, etc. The field blank preparation was identical to the sample preparation except that the Kim wipes did not contact with the window surfaces. For the analysis of total organic carbon (TOC), an approximate $0.01 \mathrm{~m}^{2}$ area of the window surfaces were simultaneously wiped with DCM-wetted quartz microfiber filters. Before sampling, the microfiber filter was pretreated in a muffle furnace at $450{ }^{\circ} \mathrm{C}$ overnight and then wrapped with aluminum foil until analysis. Organic carbon was not detected in blank and field blank microfiber filters. After sampling, all samples and field blanks were weighted, wrapped in aluminum foil, sealed in valve bags and then stored in a refrigerator at $-20^{\circ} \mathrm{C}$.

PUF disks (diameter, $14 \mathrm{~cm}$; thickness, $1.20 \mathrm{~cm}$; surface area, $360.65 \mathrm{~cm}^{2}$; mass, $4.50 \mathrm{~g}$; volume, $184.73 \mathrm{~cm}^{3}$; density, $0.0244 \mathrm{~g} \mathrm{~cm}^{3}$; Tisch Environmental. Inc) were housed in stainless steel domed chambers. Samplers were deployed for approximately 70 days between June and August 2014 at 11 sites in offices and residential buildings in Beijing. A field blank was prepared by installing a pre-washed PUF disk to the sampler for $5 \mathrm{~min}$ and then wrapped in aluminum foil and placed in zipper bags until analysis. PUF disks were handled using solvent-rinsed tongs. Sampling chambers were prewashed and solvent-rinsed with acetone prior to installation of the passive sampling media. PUF disks were extracted by ASE 350 with a 1:1 (volume to volume) mixture of dichloromethane and hexanes prior to use.

\subsection{Sample pretreatment, instrument analysis and quantification}

The pretreatment and instrumental analysis of the SCCPs were based on our previous work with some minor modifications (Zeng et al., 2011). Briefly, each Kim wipe sample was cut into fragments, mixed with $15 \mathrm{~g}$ anhydrous sodium sulfate and then spiked with $1.0 \mathrm{ng}$ surrogate standard ${ }^{13} \mathrm{C} 10$-trans-chlordane for accelerated solvent extraction (ASE). The extract was concentrated to approximate $1 \mathrm{~mL}$ using rotary evaporation. The extract was then cleaned and fractionated on a $1.5-\mathrm{cm}$ Silica-Florisil composite column packed from the bottom to top with $3 \mathrm{~g}$ of Florisil, $2 \mathrm{~g}$ of neutral silica gel, $5 \mathrm{~g}$ of acid silica gel (30\%) and $4 \mathrm{~g}$ anhydrous sodium sulfate. The column was conditioned with $50 \mathrm{~mL}$ of $\mathrm{n}$ hexane and the sample was eluted with $40 \mathrm{~mL}$ of $\mathrm{n}$-hexane (the first fraction contained PCBs and toxaphenes), followed by $50 \mathrm{~mL}$ of dichloromethane and $50 \mathrm{~mL}$-hexane (the second fraction con- 
tained CPs and a fraction of $\mathrm{HCHs}$ ). The second fraction was concentrated to approximately $2 \mathrm{~mL}$ with Rotary evaporation and further concentrated to near dryness under a gentle stream of $N_{2}$. The fraction was then reconstituted in $200 \mu \mathrm{L}$ of cyclohexane. Prior to GC-MS analysis, $10 \mathrm{ng}$ of $\varepsilon$-HCH was added as a recovery internal standard to determine the instrumental recoveries.

All analyses were performed using high-resolution gas chromatography/electron capture negative ion low-resolution mass spectrometry (HRGC/ECNI-LRMS) with a 7890A gas chromatograph coupled with an Agilent 7000B Triple Quad MS. An aliquot of $1 \mu \mathrm{L}$ of the final extract was introduced into a DB-5MSUI (30 m length, $0.25 \mathrm{~mm}$ i.d., $0.25 \mu \mathrm{m}$ film thickness) capillary column by pulsed splitless injection. The injector temperature was $280{ }^{\circ} \mathrm{C}$. Helium gas was used as the carrier gas at a constant flow of $1.2 \mathrm{~mL} / \mathrm{min}$, and methane was the reagent gas. The oven temperature program for the chromatographic separation was as follows: 1 min isothermal at $100{ }^{\circ} \mathrm{C}$, increased to $160{ }^{\circ} \mathrm{C}$ at $30{ }^{\circ} \mathrm{C} / \mathrm{min}$, held for $5 \mathrm{~min}$, then ramped to $310{ }^{\circ} \mathrm{C}$ at $30{ }^{\circ} \mathrm{C} / \mathrm{min}$ and finally held for $17 \mathrm{~min}$. The transfer line temperature and ion source temperature were set to $280{ }^{\circ} \mathrm{C}$ and $200{ }^{\circ} \mathrm{C}$, respectively. Selected ion monitoring (SIM) mode was used under $\mathrm{NCI}$ conditions, and the most abundant and second most abundant isotope ions of $[\mathrm{M}-\mathrm{Cl}]^{-}$for each congener group were selected as the quantification and confirmation ions, respectively. Retention windows were optimized for each monitored ions. Our targeted SCCP congeners $(\mathrm{C} 10-\mathrm{C} 13)$ were those containing 5-10 chlorine atoms. The quantification of the SCCPs was conducted by four separate injections according to the optimized conditions described in our previous work (Zeng et al., 2011).

Total organic carbon was analyzed using a thermal-optical carbon analyzer (Elementar Vario TOC, Elementar, Germany). Each sample was pretreated with $1 \mathrm{~mol} / \mathrm{L} \mathrm{HCl}$ to remove carbonate residues for the TOC measurements.

\section{3. $Q A / Q C$}

To avoid possible contaminants, all glassware was soaked in deionized water with Decon 90, which is a neutral wash solution designed for organic matter, for over $6 \mathrm{~h}$, and then washed three times with deionized water. The glassware was then baked in an oven overnight at $180{ }^{\circ} \mathrm{C}$. Sodium sulfate was heated to $450{ }^{\circ} \mathrm{C}$ overnight prior to use. Each batch of 5-8 samples included one procedural blank. In total, procedural and field blanks were under the instrumental detection limit of $0.25 \mathrm{ng}$. Thus, the final reported data in this study were not blank corrected. The method detection limit is defined as three times the standard deviation of procedural blanks from all of the batches. The method detection of limit (MDL) was $0.55 \mathrm{ng}$. The recoveries of spiked SCCP standards in precleaned Kim Wipes were in the range $71 \%-110 \%$. The internal standard $\left({ }^{13} \mathrm{C}\right.$-trans-chlordane) recoveries of organic film samples were in the range of 53\%-122\% (Table S2.). The recoveries of spiked SCCP in blank PUF ranged from $64 \%$ to $105 \%$. The internal standard recoveries were between $57 \%$ and $95 \%$ for PUF samples.

In this study, in order to further evaluate the possible interference of PCBs and toxaphenes to the SCCPs during the quantitation, a high resolution GC-QTOF-EI-MS was also applied. The results indicated no PCBs were found in the samples after clean up procedures for this particular matrix. As for toxaphene, the results in another research group in our institute which is conducting research on toxaphene levels in the environment indicated that except for specific contaminated sites such as historical toxaphene manufacturing facilities, the environmental levels were always under the LOD in other sites in China.

As for the interference from MCCP and LCCPs, no LCCPs were detected under NCI mode at a concentration of $10 \mathrm{ng} / \mu \mathrm{L}$. In order to minimize the interferences of MCCPs, we carefully deter- mined the retention time range of each congener group and calculated their ratios of the quantitative ion and qualitative ion based on the chromatographic and mass spectrometric results using SCCPs and MCCPs standards. The specific procedures are based on the response ratio of quantification ion and qualification ion to determine the percentage of SCCPs contribution to the total response of a certain $m / z$. This quantification method in this work can thus further reduce the interferences of MCCPs (Zeng et al., 2011).

We participate in international interlab comparisons on SCCP analysis and obtained a result below the reference value by $50 \%$. According to (Sverko et al., 2012) LRMS method can generate a $300 \%$ deviation from those of HRMS, therefore the result obtained in our lab is relatively reliable. Data analysis was performed with the IBM SPSS Statistics 19 and Origin Pro 8 software packages.

\section{Results and discussion}

\subsection{SCCPs concentrations and formula groups in the organic films}

Sampling area and mass D-value are both recorded. We tried to calculate the concentrations of SCCPs in the organic films based on the mass fraction. Unfortunately, the total mass for each sample is relatively small and if calculated using mass fraction, the deviation of the results is relatively bigger. As area-normalized results are also commonly used by other studies on PBDE, PAHs etc. (Butt. et al., 2004; Pan et al., 2012). Therefore, the SCCP concentrations (SI Table S2) in this study are presented as area-normalized concentrations. Fig. 2 shows the paired area-normalized concentrations of the total SCCPs from 19 sampling sites across Beijing. The concentration of total SCCPs ( $\Sigma$ SCCPs) on the indoor glass surfaces ranged from $0.34 \mu \mathrm{g} / \mathrm{m}^{2}$ to $54 \mu \mathrm{g} / \mathrm{m}^{2}$ (GM: $5.9 \mu \mathrm{g} / \mathrm{m}^{2}$ ), and the corresponding concentrations on the outside glass surfaces were in the range of $0.48 \mu \mathrm{g} / \mathrm{m}^{2}$ to $114 \mu \mathrm{g} / \mathrm{m}^{2}$ (GM: $3.35 \mu \mathrm{g} / \mathrm{m}^{2}$ ). On the whole, $\Sigma$ SCCPs on the indoor side were higher than that on the outside but not significantly $(p=0.06)$. However, if a few exceptionally high values (i.e., S5, S7, S9 and S10) were not included in the comparison, we found that the interior SCCPs levels were significantly higher $(p=0.02)$ than the corresponding exterior side. This is quite different from other observations for PAHs (Butt et al., 2004; Unger and Gustafsson, 2008), which implies that indoor exposure might be an important pathway of SCCPs to the general population in the sampling area. We grouped the 19 sites into three categories outdoor $>>$ indoor, indoor $\approx$ outdoor and indoor $>>$ outdoor, among them S7 and S9 outdoor $>>$ indoor, S15, S18 and S19 indoor $>>$ outdoor, the others are indoor $\approx$ outdoor

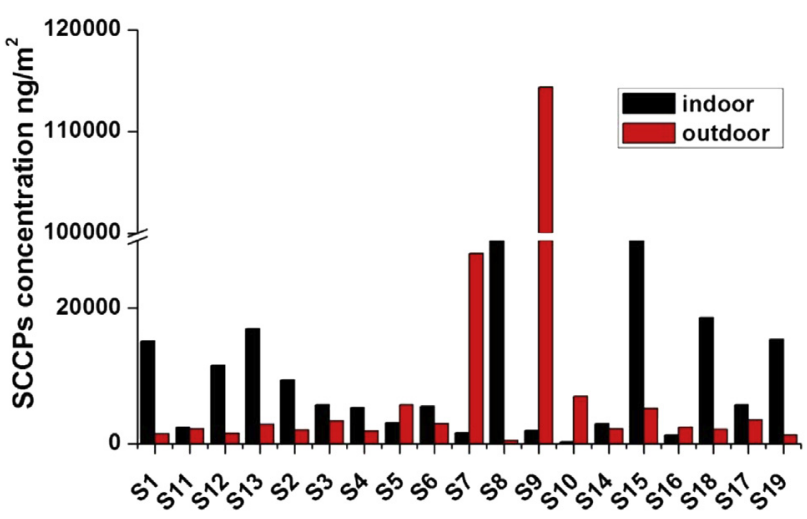

Fig. 2. SCCPs content on the indoor and outdoor window surfaces. S1: background site in suburban, Beijing; S2-S10: residential houses; S11-S13: dormitories; S14S19: offices in Beijing. 
ones. The interesting thing is S7 and S9 both are residential houses and S15, S18, and S19 are offices. The SCCPs profiles of these unusual sites were also different from other sites (SI, Fig. S4). This differentiation may suggest different sources of SCCPs (i.e., window caulking, point sources like chemical plants). Among the unusual sites, S9 is two miles away from a chemical plant and S15 is close to a main road.

Organic films on window surfaces have been thought to be formed by the deposition of atmospheric particles and condensation of gas-phase SVOCs (Law and Diamond, 1998). Usually, the organic carbon concentration in organic films is consistent with the concentrations of SVOCs (Unger and Gustafsson, 2008). In this study, the TOC contents in the organic films on the indoor window surfaces ranged from $3.68 \mathrm{mg} / \mathrm{m}^{2}$ to $172 \mathrm{mg} / \mathrm{m}^{2}$ (GM: $13.1 \mathrm{mg} / \mathrm{m}^{2}$ ), and the corresponding contents on the outdoor window surfaces were in the range of $0.37 \mathrm{mg} / \mathrm{m}^{2}-95 \mathrm{mg} / \mathrm{m}^{2}$ (GM: $15 \mathrm{mg} / \mathrm{m}^{2}$ ), which is higher than the values previously measured on window surfaces in Guangzhou and Hong Kong(Butt et al., 2004). Significant linear relationships were found between the TOC normalized $\Sigma$ SCCPs on the interior side and exterior side with TOC content ( $R=0.41, p=0.01$ and $R=0.51, p=0.01$, respectively), which implies that organic matter is a main factor that influences the SCCP concentrations in organic films. The corresponding TOC normalized $\Sigma$ SCCPs on the interior side are in the range of $63 \mu \mathrm{g} / \mathrm{g}-5.24 \mathrm{mg} / \mathrm{g}$ (GM: $472 \mu \mathrm{g} / \mathrm{g}$ ), which are higher than those measured on the outdoor surfaces (0.55-1.53 mg/g with GM: $0.19 \mu \mathrm{g} / \mathrm{g})$, except for S5, S7, S9, S10, and S17.

Fig. 3 shows the distribution of formula groups on the indoor and outdoor samples from the different sites examined. $\mathrm{C}_{10}$ and $\mathrm{C}_{11}$ congeners were found to be the most abundant carbon ho-
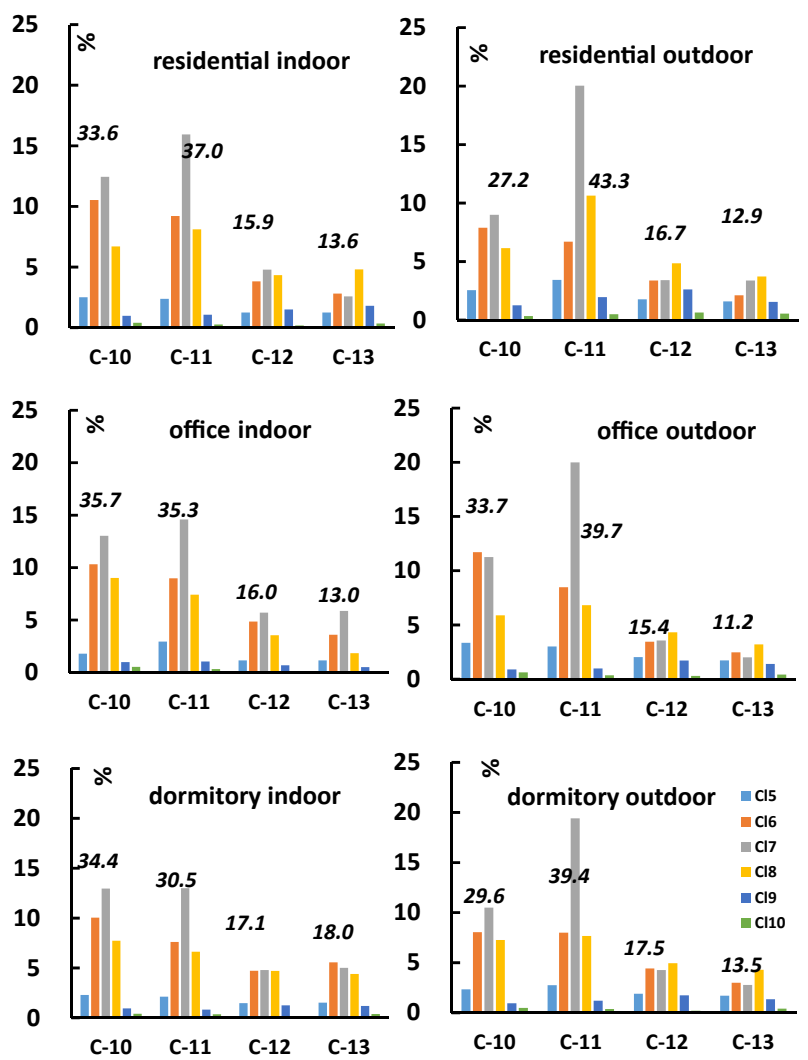

Fig. 3. Different formula group abundances (in percentages) of SCCPs (indoor/outdoor) for different site types (i.e., residential buildings, offices, and dormitories). The italic numbers indicate the percentages of each carbon chain length group. mologs, which accounted for almost $70.0 \%$ of $\Sigma$ SCCPs for both the exterior and interior films on the window surfaces. No significant differences were found for the congener patterns of SC$\mathrm{CPs}$ between the interior and exterior sides. Generally, the concentrations of the $C_{11}$ congener group exceeded that of the $C_{10}$ congener group for the outdoor samples whereas the concentrations of the $C_{10}$ and $C_{11}$ congener groups from the samples from the interior side were almost equivalent. This result consistent with the work on SCCPs from indoor air samples collected from Stockholm, Sweden and London, United Kingdom (Fridén et al., 2011; Peters et al., 2000). However, this result is different from our previous finding on the pattern of the atmospheric SCCPs in Beijing (Wang et al., 2012), where $C_{10}$ was considered to be the most predominant homolog ( $>70.0 \%$ of $\Sigma$ SCCPs). As for different chlorine atom homologs, $\mathrm{Cl}_{6}$ and $\mathrm{Cl}_{7}$ were the most abundant congener groups on both the indoor and outdoor window surfaces from the sampling sites. Therefore, the $\mathrm{C}_{11} \mathrm{Cl}_{7}$ formula group is the most abundant congener group in all of the samples, accounting for $15.1 \%$ of $\Sigma$ SCCP for the indoor samples and over $20.0 \%$ for the outdoor samples. Correspondingly, TOC normalized concentrations of the $\mathrm{C}_{11} \mathrm{Cl}_{7}$ congener group accounted for $15.4 \%$ and $18.5 \%$ of TOC normalized $\Sigma$ SCCPs, respectively. As shown in Fig. 3 outdoor side gathers more C11 (heavier) group and indoor side gathers more C10 (lighter) group. The organic films on the exterior side tended to gather heavier SCCPs (i.e., more chlorine atoms with longer carbon chain lengths) whereas the films on the interior side tended to gather lighter SCCPs (i.e., fewer chlorine atoms with shorter carbon chain lengths). In the indoor environment, SCCPs in the organic film are primarily derived from compounds in the gas-phase, whereas in the outside environment, the pollution was jointly influenced by the gas and particle phase, such as dry/wet deposition, washout, etc. Our work on the SCCPs in the atmosphere of Beijing suggested that heavier congeners of SCCPs had higher affinities to the particle phase. This observation has also been determined for the behavior of PAHs (Pan et al., 2012).

\subsection{SCCPs concentrations and formula groups in indoor air}

Indoor air SCCPs were detected in all of the 11 sites with concentrations ranging between 0.06 and $1.35 \mu \mathrm{g} / \mathrm{m}^{3}$ (GM: $0.23 \mu \mathrm{g} / \mathrm{m}^{3}$ ), which is significantly higher than the levels detected in outdoor environments in Beijing in both the winter and summer seasons (0.4-9.7 ng/ $\mathrm{m}^{3}$ in winter and $108-316 \mathrm{ng} / \mathrm{m}^{3}$ in summer) (Wang et al., 2012). The possible reasons for this increase may be because of the different sources of SCCPs between the indoor and outdoor air.

Formula group abundance profiles of indoor air (Fig. 4) showed a similar distribution pattern with our findings for the outdoor gas-phase SCCPs (Wang et al., 2012). Specifically, $\mathrm{Cl}_{6}$ was the most
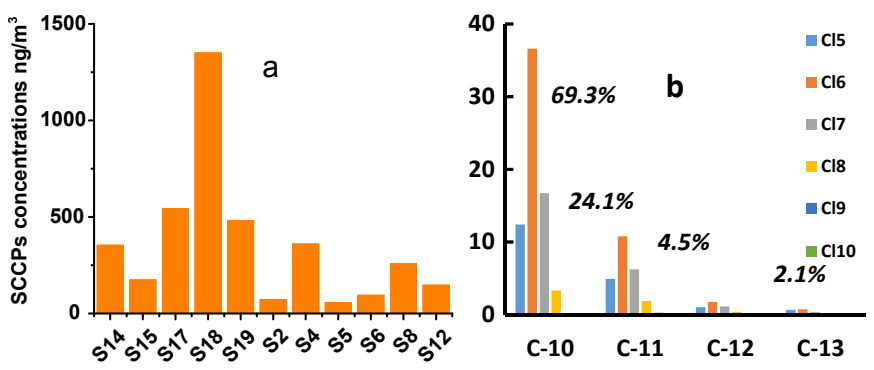

Fig. 4. $\Sigma$ SCCPs concentrations in indoor air from 11 sites (offices: S19, S15, S18, S15, S14; residential houses: S5, S4, S8, S6, S2; dormitory: S12) (a); $\Sigma$ SCCPs congener group profiles in indoor air from 11 site (b). 
abundant chlorine atom congener and $\mathrm{C}_{10}$ accounted for almost $70.0 \%$ of SCCPs. However, the $\mathrm{C}_{12-13}$ congener group contributed only $3.0 \%$ of $\Sigma$ SCCPs to the indoor air samples.

\section{3. $\Sigma S C C P_{S}$ from different sampling site types}

A study (Duigu et al., 2009) on the relationship between the building characteristics and the composition of the surface films showed that the building characteristics significantly affect the accumulation of pollutants on the window's surface. Overall, the results in this study showed differences of $\Sigma$ SCCPs in the organic film among three different building types (i.e., dormitories, residential houses and offices). For the indoor side, $\Sigma$ SCCPs (GM $23.2 \mu \mathrm{g} / \mathrm{m}^{2}$ ) in the window films from the offices were higher than those from the dormitories and residential rooms; specifically, as shown in Fig. 5, on the interior side, O (averaged at $23.2 \mu \mathrm{g} / \mathrm{m}^{2}$ ) > D (averaged at $10.3 \mu \mathrm{g} / \mathrm{m}^{2}$ ) > R (averaged at $3.1 \mu \mathrm{g} / \mathrm{m}^{2}$ ); on the exterior side, $\mathrm{R}$ (averaged at $0.6 \mu \mathrm{g} / \mathrm{m}^{2}$ ) > 0 (averaged at $2.8 \mu \mathrm{g} / \mathrm{m}^{2}$ ) > D (averaged at $2.2 \mu \mathrm{g} / \mathrm{m}^{2}$ ), where $\mathrm{D}, \mathrm{O}$, and $\mathrm{R}$ indicate dormitory, office, and residential, respectively.

Although no significant difference was found between the different site types for the indoor air SCCPs, SCCPs levels tended to be higher in the offices than the residential buildings and dormitories (Fig. 3).

The direction of the window face and its height were also considered during sampling. We collected samples from different floors within each building at S11, S12, and S13 to examine the influence of height on the distribution of the SCCPs. The heights range from 13.32 to $43.52,13.30$ to 35.00 , and 5.87 to $33.98 \mathrm{~m}$ at sites S11, S12, and S13, respectively. The SCCP content and pattern distributions were uneven and no significant trend was found. The reason for this might be that the distribution of the SCCPs was uniform in the air over the relatively small range of height we investigated. Different window faces offer distinct micro-climates because of their variable exposure to sunrays, wind, and precipitation. However, no difference was observed for the four distinct window faces, which is likely to be a result of the limited number of samples that were measured and SCCPs are a class of chemicals that are not photosensitive.

\subsection{Estimating the film-air partitioning of SCCPS}

Organic compounds in the air can be partitioned into the organic phase in the organics. For this reason, organic film levels

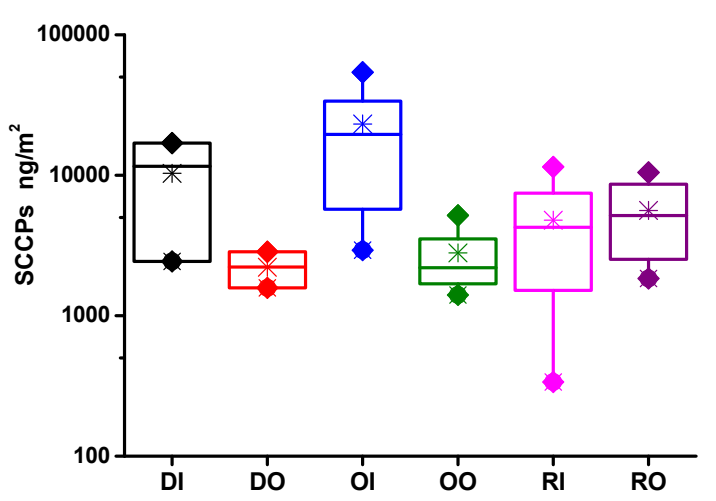

Fig. 5. A box-plot of the indoor/outdoor $\Sigma$ SCCPs concentration of film samples from different site types (DI for dormitory indoor, DO for dormitory outdoor, OI for office indoor, $\mathrm{OO}$ for office outdoor, RI for residential indoor, RO for residential outdoor). Individual samples are shown as diamonds. The range of each box represents the 25th and 75th percentile, whereas the whiskers represent the 10th and 90th percentile. Maximum and minimum levels are marked with " $x$ ". of organic pollutants might act as indicators for the indoor atmospheric exposure level of POPs for the general public. According to $\mathrm{Li}$ et al., the growth rate of organic film tend to be constant after a fast growing period (Li et al., 2010) and this growth rate is related to the Koa value of the targets (Cetin and Odabasi, 2011). The Koa value of SCCPs is similar to that of the PBDEs and PCBs and the Li et al., showed that the growth rate tend to be constant after 40-60 d. The windows sampled in this work were not washed for at least three months. Thus, the growth rates tend to be consistent under various wind speed (Csiszar et al., 2012) and we can assume an equilibrated partition of the SCCPs in the gas-phase in the indoor air and the organic films. The concentrations and congener group distributions of the SCCPs in indoor air from 11 sampling sites based on PAS are provided in Fig. S3 (SI). According to the work of (Unger and Gustafsson, 2008) the film-air partitioning of SCCPs can be described with the following equation:

$\mathrm{PC}_{\mathrm{air}}=\mathrm{C}_{\mathrm{om}} / \mathrm{K}_{\mathrm{om}-\text { air }}(\mathrm{T})$,

where $\mathrm{PC}_{\mathrm{air}}, \mathrm{C}_{\mathrm{OM}}$, and $\mathrm{K}_{\mathrm{om} \text {-air }}$ represent the predicted SCCP concentration in air, the SCCP concentration in organic matter, and the partition coefficient between organic matter and air, respectively. $\mathrm{C}_{\mathrm{OM}}$ can be estimated through TOC normalized concentrations based on a factor of 1.6 (Li et al., 2010), which is a ratio based on the average molecular weight in organic aerosols. $\mathrm{K}_{\text {om-air }}$ can be estimated using a series of physico-chemical properties of SC$\mathrm{CPs}$, including the octanol-air partitioning coefficient $\left(\mathrm{K}_{\mathrm{oa}}\right)$ and the octanol-water partition coefficient $\left(\mathrm{K}_{\mathrm{ow}}\right)$. Because of the lack of experimental data for $\mathrm{K}_{\mathrm{oa}}$ and $\mathrm{K}_{\mathrm{ow}}$, we calculated the SCCP properties using the physical-chemical estimation models from the Estimation Programs Interface suite of programs (Wang et al., 2012, 2015). The average indoor environmental temperature $\left(26^{\circ} \mathrm{C}\right)$ during the sampling period was close to the referenced temperature $\left(25^{\circ} \mathrm{C}\right)$ used for the calculations. Specific structures were needed to calculate the physico-chemical properties. However, it is not possible to perform such calculations for each isomer. For this reason, we choose four isomers from each molecular formula group. The four isomers cover different distributions of chlorine atoms on the carbon chain: one chlorine atom at each end, in the middle of the carbon chain, equally distributed along the entire length of the carbon chain, and at one end of the carbon chain. Fig. S1 shows the minimum, medium and max log Koa values for the different formula groups. To examine the air-film partition, we plotted the logarithm ratio of the concentrations in the organic film $\left(C_{f}\right)$ and air $\left(C_{a}\right)(\log$ $\mathrm{C}_{\mathrm{f}} / \mathrm{C}_{\mathrm{a}}$ ) against $\log \mathrm{K}_{\mathrm{oa}}$. This analysis showed a strong linear relationship ( $R=0.67, p=0.01$ ) (Fig. S3a). According to McLachlan's three-process theory (Mclachlan, 1999), the type of positive slope for log Koa 9-11 indicates a kinetically limited gaseous deposition. Single formula group contents were also compared to $\Sigma$ SCCPs in the window surface film and in air. Because the $\mathrm{C}_{10} \mathrm{Cl}_{7}$ congener group comprised a large fraction of contaminants in both the film and air samples, we chose it to interpret the film-to-air concentration of SCCPs. The calculated $\log$ Koa for the $\mathrm{C}_{10} \mathrm{Cl}_{7}$ formula group are in the range of 9-11. Correlation analysis indicated the concentrations of the $\mathrm{C}_{10} \mathrm{Cl}_{7}$ formula group in both the organic film and air possessed significant linearity with $\mathrm{Ca}(R=0.77, p=0.01$; $R=0.97, p=0.01$ ) (Fig. S3b). This linearity implies that the $\mathrm{C}_{10} \mathrm{Cl}_{7}$ film concentration may act as an indicator for long term atmospheric $\Sigma$ SCCPs levels.

Organic matter on window glass surfaces can act as lipid-like matrices; if we consider the density of octanol $\left(\rho_{\text {oct }}=0.8275\right)$, the partition trend between organic matter and water (Nisheeth et al., 1999; Escher et al., 2000; Schwarzenbach et al., 2003a) can be described as:

$\log \mathrm{K}_{\mathrm{OM} \text {-water }}(298)=\left(0.91 \mathrm{Log} \mathrm{K}_{\mathrm{ow}}\right) / \rho \mathrm{oct}+0.50$. 


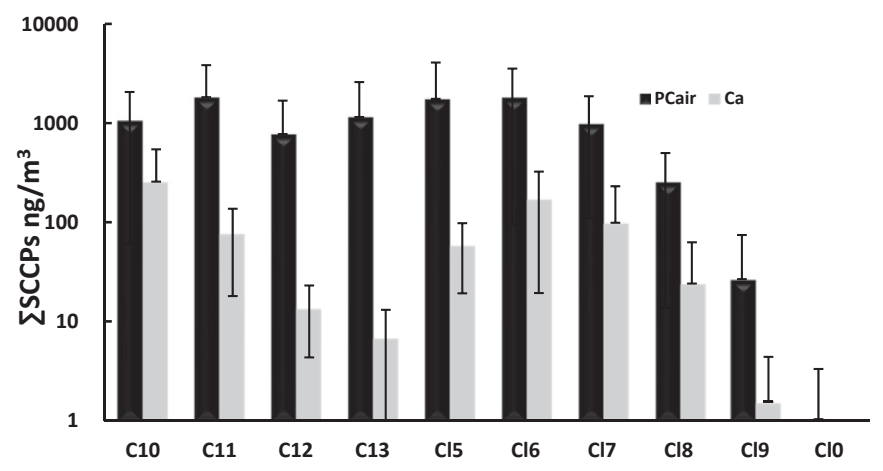

Fig. 6. Comparison of air concentrations of SCCP congener groups of different carbon chain lengths and chlorine atoms predicted from window film content $\left(\mathrm{PC}_{\mathrm{air}}\right)$ and obtained with polyurethane form materials (PUF) high-volume samplers (Ca) in Beijing.

Then, the partition coefficient between organic matter and air (Schwarzenbach et al., 2003b) can be determined using:

$\mathrm{K}_{\mathrm{OM}-\mathrm{air}}(298)=\mathrm{K}_{\mathrm{OM}-\text { water }} / \mathrm{K}_{\mathrm{aw}}$

$\mathrm{K}_{\mathrm{OM} \text {-air }}$ values for 24 formula groups of SCCPs are provided in Fig. S2. Based on eqs. (1)-(3), PC $_{\text {air }}$ was calculated and compared with the SCCP concentrations in the atmosphere obtained by indoor PAS at 11 concurrent sites. The air volume was calculated using an adaptation of the equivalent volume method (Harner et al., 2004). As shown in Fig. 6, the organic-film-interpreted $\mathrm{PC}_{\text {air }}$ value overestimates the gas-phase SCCPs $C_{a}$ measured by PAS. A key reason for this might be that the chosen $\mathrm{K}_{\mathrm{OA}}$ values representing each formula group do not reflect the characteristics of all of the isomers, which may vary within each group. Moreover, window surfaces also accumulate aerosols from the atmosphere (Diamond et al., 2000; Gingrich et al., 2001), in addition to adsorbing gasphase SCCPs.

\section{Conclusions}

In this study, high concentrations of SCCPs were observed in organic films on both interior and exterior window surfaces which suggest possible relatively high exposure for human beings in daily life. The concentrations of SCCPs in indoor samples were usually higher than those in outdoor samples. In general, light-weight SCCPs tend to gather in interior window films than in exterior window films while heavy-weight SCCPs tend to gather on the exterior side. This observation is compatible with the found of PAHs that indoor environment is a possible source for light-weight contaminants. The higher levels of SCCPs in indoor samples indicated that indoor decorations might be an important source for SCCPs except from the outdoor air as the major source of pollution.

\section{Acknowledgments}

We thank the National Basic Research Program of China (2015CB453100), the National Natural Science Foundation of China (21477141, 21222702, 21321004), and the Strategic Priority Research Program of the Chinese Academy of Science (XDB14010400, YSW2013B01) for financial support.

\section{Appendix A. Supplementary data}

Supplementary data related to this article can be found at http: //dx.doi.org/10.1016/j.chemosphere.2015.09.075.

\section{References}

Butt, C.M., Diamond, M.L., Truong, J., 2004. Spatial distribution of polybrominated diphenyl ethers in Southern Ontario as measured in indoor and outdoor window organic films. Environ. Sci. Technol. 38, 724-731.

Cetin, B., Odabasi, M., 2011. Polybrominated diphenyl ethers (PBDEs) in indoor and outdoor window organic films in Izmir, Turkey. J. Hazard. Mater. 185, 784-791.

Chen, M.Y., Luo, X.J., Zhang, X.L., He, M.J., Chen, S.J., Mai, B.X., 2011. Chlorinated paraffins in sediments from the Pearl River Delta, South China: spatial and temporal distributions and implication for processes. Environ. Sci. Technol. 45 9936-9943.

Csiszar, S.A., Diamond, M.L., Thibodeaux, L.J., 2012. Modeling urban films using a dynamic multimedia fugacity model. Chemosphere 87, 1024-1031.

De Boer, J., 2010. The Handbook of Environmental Chemistry. Springer, Berlin Heidelberg, Germany.

Diamond, M.L., Gingrich, S.E., Fertuck, K., McCarry, B.E., Stern, G.A., Billeck, B., Grift, B., Brooker, D., Yager, T.D., 2000. Evidence for organic film on an impervious urban surface: characterization and potential teratogenic effects. Environ. Sci. Technol. 34, 2900-2908.

Duigu, J.R., Ayoko, G.A., Kokot, S., 2009. The relationship between building characteristics and the chemical composition of surface films found on glass windows in Brisbane, Australia. Build. Environ. 44, 2228-2235.

Escher, B.I., Schwarzenbach, R.P., Westall, J.C., 2000. Evaluation of liposome-wate partitioning of organic acids and bases. 1. Development of a sorption model. Environ. Sci. Technol. 34, 3954-3961.

European Commission, 2000. Directive 2000/60/EC of the European Parliament and of the Council of 23 October 2000 establishing a framework for Community action in the field of water policy. Off. J. Eur. Commun. P 001-0073.

Feo, M.L., Eljarrat, E., Barcelo, D., 2009. Occurrence, fate and analysis of polychlorinated n-alkanes in the environment. Trends Anal. Chem. 28, 778-791.

Fridén, U.E., McLachlan, M.S., Berger, U., 2011. Chlorinated paraffins in indoor air and dust: concentrations, congener patterns, and human exposure. Environ. Int. 37, 1169-1174.

Gingrich, S.E., Diamond, M.L., Stern, G.A., 2001. Atmospherically derived organic surface films along an urban-rural gradient. Environ. Sci. Technol. 35, 4031-4037.

Harner, T., Shoeib, M., Diamond, M., Stern, G., Rosenberg, B., 2004. Using passive air samplers to assess urban-rural trends for persistent organic pollutants. 1 Polychlorinated biphenyls and organochlorine pesticides. Environ. Sci. Technol. 38, 4474-4483.

Krogseth, I.S., Breivik, K., Arnot, J.A., Wania, F., Borgena, A.R., Schlabacha, M., 2013. Evaluating the environmental fate of short-chain chlorinated paraffins (SCCPs) in the Nordic environment using a dynamic multimedia model. Environ. Sci. Process. Impacts 15, 2240-2251.

Lam, B., Diamond, M.L., Simpson, A.J., Makar, P.A., Truong, J.,N., HernandezMartinez, A., 2005. Chemical composition of surface films on glass windows and implications for atmospheric chemistry. Atmos. Environ. 39, 6578-6586.

Law, N.L., Diamond, M.L., 1998. The role of organic films and the effect on hydrophobic organic compounds in urban areas: a hypothesis. Chemosphere 36, 2607-2620.

Li, J., Lin, T., Pan, S.H., Xu, Y., Liu, X., Zhang, G., Li, X.D., 2010. Carbonaceous matter and PBDEs on indoor/outdoor glass window surfaces in Guangzhou and Hong Kong, South China. Atmos. Environ. 44, 3254-3260.

Liu, Q.T., Chen, R., Mccarry, B.E., Diamond, M.L., Bahavar, B., 2003a. Characterization of polar organic compounds in the organic film on indoor and outdoor glass windows. Environ. Sci. Technol, 37, 2340-2349.

Liu, Q.T., Diamond, M.L., Gingrich, S.E., Ondov, J.M., Maciejczyk, P., Stern, G.A., 2003b. Accumulation of metals, trace elements and semi-volatile organic compounds on exterior window surfaces in Baltimore. Environ. Pollut. 122, 51-61.

Ma, X.D., Zhang, H.J., Zhou, H.Q., Na, G.S., Wang, Z., Chen, C., Chen, J.W., Chen, J.P., 2014. Occurrence and gas/particle partitioning of short- and medium-chain chlorinated paraffins in the atmosphere of Fildes Peninsula of Antarctica. Atmos. Environ. 90, 10-15.

Mclachlan, M.S., 1999. Framework for the interpretation of measurements of SOCs in plants. Environ. Sci. Technol. 33, 1799-1804.

, 2000, In the Handbook of Environmental Chemistry. In: Muir, D.C.G., Stern, G.A. Tomy, G.T., Paasivirta, J. (Eds.). Springer, Heidelberg, Berlin, New York, p. 203.

Nisheeth, P.B., Wan, Y.S., David, G.B., Donald, M., 1999. Tricaprylin-water partition coefficients and their temperature dependence for selected chlorobenzenes. J. Chem. Eng. Data 44, 40-43.

Pan, S.H., Li, J., Lin, T., Zhang, G., Li, X.D., Yin, H., 2012. Polycyclic aromatic hydrocarbons on indoor/outdoor glass window surfaces in Guangzhou and Hong Kong, south China. Environ. Pollut. 169, 190-195.

Peters, A.J., Tomy, G.T., Jones, K.C., Coleman, P., Stern, G.A., 2000. Occurrence of C10 C13 polychlorinated n-alkanes in the atmosphere of the United Kingdom. Atmos. Environ. 34, 3085-3090.

Schwarzenbach, R.P., Gschwend, P.M., Imboden, D.M., 2003a. Sorption II: partitioning to living media-partitioning to defined biomedia. Environmental Organic Chemistry. Wiley-Interscience, Hoboken, pp. 335-348.

Schwarzenbach, R.P., Gschwend, P.M., Imboden, D.M., 2003b. Appendix C. Environmental Organic Chemistry. Wiley-Interscience, Hoboken, pp. 1197-1208. 
Sverko, E., Tomy, G.T., Marvin, C.H., Muir, D.C.G., 2012. Improving the quality of environmental measurements on short chain chlorinated paraffins to support global regulatory efforts. Environ. Sci. Technol. 46, 4697-4698.

Tomy, G.T., Fisk, A.T., Westmore, J.B., Muir, D.C.G., 1998. Volatility of chlorinated nalkanes (C10-C12): vapor pressures and Henry's law constants. Rev. Environ. Contam. Toxicol. 17, 1252-1260.

Unger, M., Gustafsson, O., 2008. PAHs in Stockholm window films: evaluation of the utility of window film content as indicator of PAHs in urban air. Atmos. Environ. $42,5550-5557$

Van Mourik, L.M., Leonards, P.E.G., Gaus, C., Boer, J., 2015. Recent developments in capabilities for analysing chlorinated paraffins in environmental matrices: a review. Chemosphere 136, 259-272.

Wang, T., Han, S.L., Yuan, B., Zeng, L.X., Li, Y.M., Wang, Y.W., Jiang, G.B., 2012 Summer-winter concentrations and gas-particle partitioning of short chain chlorinated paraffins in the atmosphere of an urban setting. Environ. Pollut. 171, $38-45$
Wang, T., Yu, J.C., Han, S.L., Wang, Y.W., Jiang, G.B., 2015. Levels of short-chain chlorinated paraffins in pine needles and bark and their vegetation-air partitioning in urban areas. Environ. Pollut. 196, 309-312.

Wu, R.W., Harner, T., Diamond, M.L., Wilford, B., 2008. Partitioning characteristics of PCBs in urban surface films. Atmos. Environ. 42, 5696-5705.

Yuan, B., Wang, T., Zhu, N.L., Zhang, K.G., Zeng, L.X., Fu, J.J., Wang, Y.W., Jiang, G.B., 2012. Short chain chlorinated paraffins in Mollusks from Costal waters in the Chinese Bohai Sea. Environ. Sci. Technol. 46, 6489-6496.

Zeng, L.X., Wang, T., Han, W.Y., Yuan, B., Liu, Q., Wang, Y.W., Jiang, G.B., 2011. Spatial and vertical distribution of short chain chlorinated paraffins in soils from wastewater irrigated Farmlands. Environ. Sci. Technol. 45, 21002106. 